\title{
An impedance method for spatial sensing of 3D cell constructs - towards applications in tissue engineering
}

Canali, Chiara; Mazzoni, Chiara; Larsen, Layla Bashir; Heiskanen, Arto; Martinsen, Ø.G.; Wolff, Anders; Dufva, Martin; Emnéus, Jenny

\section{Published in:}

Analyst

Link to article, DOI:

10.1039/c5an00987a

Publication date:

2015

Document Version

Publisher's PDF, also known as Version of record

Link back to DTU Orbit

Citation (APA):

Canali, C., Mazzoni, C., Larsen, L. B., Heiskanen, A., Martinsen, Ø. G., Wolff, A., Dufva, M., \& Emnéus, J. (2015). An impedance method for spatial sensing of 3D cell constructs - towards applications in tissue engineering. Analyst, 140(17), 6079-6088. https://doi.org/10.1039/c5an00987a

\section{General rights}

Copyright and moral rights for the publications made accessible in the public portal are retained by the authors and/or other copyright owners and it is a condition of accessing publications that users recognise and abide by the legal requirements associated with these rights.

- Users may download and print one copy of any publication from the public portal for the purpose of private study or research.

- You may not further distribute the material or use it for any profit-making activity or commercial gain

- You may freely distribute the URL identifying the publication in the public portal 


\section{Analyst}

\section{PAPER}

View Article Online

View Journal

\section{An impedance method for spatial sensing of 3D cell constructs - towards applications in tissue engineering $\dagger$}

Cite this: DOI: 10.1039/c5an00987a

\author{
C. Canali, ${ }^{a}$ C. Mazzoni, ${ }^{a}$ L. B. Larsen, ${ }^{a}$ A. Heiskanen, ${ }^{a} \varnothing$. G. Martinsen, ${ }^{\text {b.c }}$ A. Wolff, ${ }^{a}$ \\ M. Dufva ${ }^{a}$ and J. Emnéus*a
}

Received 15th May 2015 Accepted 9th July 2015

DOI: $10.1039 / c 5 a n 00987 a$

www.rsc.org/analyst

\begin{abstract}
We present the characterisation and validation of multiplexed 4-terminal (4T) impedance measurements as a method for sensing the spatial location of cell aggregates within large three-dimensional (3D) gelatin scaffolds. The measurements were performed using an array of four rectangular chambers, each having eight platinum needle electrodes for parallel analysis. The electrode positions for current injection and voltage measurements were optimised by means of finite element simulations to maximise the sensitivity field distribution and spatial resolution. Eight different $4 \mathrm{~T}$ combinations were experimentally tested in terms of the spatial sensitivity. The simulated sensitivity fields were validated using objects (phantoms) with different conductivity and size placed in different positions inside the chamber. This provided the detection limit (volume sensitivity) of $16.5 \%$, i.e. the smallest detectable volume with respect to the size of the measurement chamber. Furthermore, the possibility for quick single frequency analysis was demonstrated by finding a common frequency of $250 \mathrm{kHz}$ for all the presented electrode combinations. As final proof of concept, a high density of human hepatoblastoma (HepG2) cells were encapsulated in gelatin to form artificial 3D cell constructs and detected when placed in different positions inside large gelatin scaffolds. Taken together, these results open new perspectives for impedance-based sensing technologies for non-invasive monitoring in tissue engineering applications providing spatial information of constructs within biologically relevant 3D environments.
\end{abstract}

\section{Introduction}

The membranes of biological cells are primarily composed of phospholipid bilayers embedding cholesterol and proteins. Hence, they are dielectrics with bound charges, displaying high resistivity (tens of $\mathrm{k} \Omega \mathrm{cm}^{2}$ ) and capacitance $\left(1 \mu \mathrm{F} \mathrm{cm} \mathrm{cm}^{-2}\right)$. Cell membranes surround a conductive aqueous environment (cytosol) consisting of a mixture of small molecules and biological macromolecules. In biological tissues, cells are surrounded by proteinaceous hydrogel-like extra cellular matrix (ECM), which combines the cellular passive electrical properties with free charges of varying mobility. Hence, under the influence of an alternating electric field, cells and tissues

\footnotetext{
${ }^{a}$ Department of Micro- and Nanotechnology, Technical University of Denmark, 2800, Kgs. Lyngby, Denmark. E-mail: jenny.emneus@nanotech.dtu.dk

${ }^{b}$ Department of Physics, University of Oslo, Sem Salands vei 24, Fysikkbygningen, 0371 Oslo, Norway

${ }^{c}$ Department of Biomedical and Clinical Engineering, Oslo University Hospital, 0372 Oslo, Norway

$\dagger$ Electronic supplementary information (ESI) available. See DOI: 10.1039/ c5an00987a
}

exhibit complex behaviour, which varies depending on the frequency range. ${ }^{1-3}$ Accordingly, electrical impedance spectroscopy (EIS) has been successfully applied as a non-invasive and cost-effective method for studying the physico-chemical properties of different biological materials in research spanning from cell biology ${ }^{4,5}$ and tissue engineering ${ }^{6,7}$ to physiology and medical technology. ${ }^{8}$

Although biophysical studies of cells in suspension have provided information on their electrical properties, ${ }^{9}$ presently the most widely used application of EIS is related to studies on $2 \mathrm{D}$ cultures of adherent cells as originally proposed by Giæver and Keese. ${ }^{10}$ 2-terminal (2T) impedance measurements, where the dielectric properties of cells modulate the interface impedance of electrodes, have been demonstrated to provide information on cell size, morphology, adhesion, spreading, proliferation and death.

In the last decade, there has been an increasing demand for cell culture models to bridge the gap between conventional $2 \mathrm{D}$ cultures and tissue engineering to better mimic the in vivo environment in terms of physiological and biomechanical behaviour. ${ }^{11,12}$ Cells cultured in a three-dimensional (3D) environment significantly differ in terms of cell-cell and cell- 
matrix interactions from those grown in conventional 2D cultures. They acquire an in vivo-like morphology that better reflects the mechanisms of proliferation, survival, differentiation and migration. ${ }^{13}$ Such dynamics may be difficult to monitor using conventional microscopy techniques due to difficulties with light penetration and scattering effects in thick scaffolds. A promising tool in this regards is EIS detection, which has been demonstrated for monitoring thin 3D cultures. ${ }^{14}$ However, when increasing the dimensions of the 3D matrix towards the requirements of tissue engineering, the developed sensing method has to provide sufficient spatial resolution for mapping cell distribution. We have recently demonstrated EIS detection in large 3D cell cultures using complementary $2 \mathrm{~T}$ - and $3 \mathrm{~T}$ measurements to collect spatially distributed information within the $3 \mathrm{D}$ space. ${ }^{6}$

Further development of EIS monitoring in large scaffolds relevant for tissue engineering is, however, necessary to improve the achieved spatial resolution. Inspiration can be found in physiological impedance measurements on tissues and organs, usually defined as bioimpedance. ${ }^{15}$ These measurements give an insight into the composition, i.e. density and integrity, of tissues and organs, correlating the results to pathophysiological processes. More recently, electrical impedance tomography (EIT) has emerged as a suitable technique for imaging organs, e.g., brain and breast, as well as their activity, e.g., lung ventilation and gastric empting. ${ }^{16}$ In EIT, four-terminal (4T) impedance measurements are commonly employed to minimise errors due to electrode interface impedance $\left(e . g \text {. polarisation impedance, } Z_{\mathrm{p}}\right)^{17,18}$ and to maximise the reciprocity of the measurement method. ${ }^{19,20}$ Two separate electrode couples are used as current carrying (CC) and voltage pick-up (PU) electrodes. The measured impedance is the transfer impedance, ${ }^{21}$ i.e. a part of the excitation signal carried by the CC couple is transferred to the PU couple depending on the material properties and composition. Modern instrumentation, having high input impedance, allows measurements in which the PU electrodes are not current carrying, eliminating the contribution of the PU electrode interfaces to the measured impedance. ${ }^{6,22,23}$ Moreover, the application of an independent CC couple facilitates measurements without the sensitivity field $(S)$ being influenced by the interface impedance of the CC couple.

In this work, we present the characterisation and validation of an impedance-based method using multiplexed 4T impedance measurements for sensing the distribution of cell aggregates within large 3D gelatin scaffolds. An array of four rectangular measurement chambers, each chamber comprising eight platinum (Pt) needle electrodes positioned along the perimeter, was used for parallel analysis. Using different combinations of electrodes (modes) as the CC and PU couple, we demonstrate the potential of using multiplexed $4 \mathrm{~T}$ measurements to gather information on the spatial distribution of cell aggregates within a 3D environment. Finite element (FE) simulations were used to study the influence of electrode positioning within the measurement chamber and the resulting sensitivity field $(S){ }^{22,24}$ The approach was first validated using cylindrical metal and plastic test objects (phantoms) of varying dimensions placed in different positions to find the detection limit (volume sensitivity), i.e. the smallest detectable volume with respect to the measurement chamber size, and a common frequency for the eight sensing modes to facilitate quick single frequency analysis. Artificial cylindrical 3D cell constructs, composed of a high density of human hepatoblastoma (HepG2) cells encapsulated in gelatin, were then introduced in different positions inside a larger gelatin scaffold in each measurement chamber. The presented $4 \mathrm{~T}$ impedance sensing provides information on the spatial position of the constructs within biologically relevant $3 \mathrm{D}$ environments, opening new possibilities for non-invasive 3D monitoring in tissue engineering applications.

\section{Experimental}

\subsection{FE simulation of sensitivity field $(S)$ distribution}

FE simulations were carried out using Comsol Multiphysics v.4.4 to map the sensitivity field $(S)$ distribution for different combinations of current carrying (CC) and voltage pick-up (PU) electrodes placed within the measurement chamber. ESI $\mathrm{S} 1, \dagger$ provides details of different electrode configurations used in EIS $(2 \mathrm{~T}, 3 \mathrm{~T}, 4 \mathrm{~T})$ and the technique for calculating $S .^{25}$ Regions of positive/(negative) sensitivity are defined where the measured impedance increases/(decreases) with an increase in the actual impedance of the sample. ${ }^{26}$ It is relevant to point out that $S$ does not quantitatively reflect what is experimentally measured, but it expresses how much weight the local impedance in a specific $3 \mathrm{D}$ sub-volume has on the total measured impedance. ${ }^{25}$ Computational models were built to maximise regions of positive sensitivity, assuming the chamber was filled with a commercial conductivity standard solution with a conductivity close to that of physiological solutions $\left(\sigma=1.3 \mathrm{~S} \mathrm{~m}^{-1}\right.$ and $\left.\varepsilon_{\mathrm{r}}=80\right)$. The mesh consisted of 153891 tetrahedral elements with an average element quality of 0.672. Eight different combinations of electrode pairs were optimised (Fig. 1Aa-h) to focus on the sensitivity field distribution in specific sub-volumes within the whole measurement chamber volume (Fig. 1Ba-h). A potential of $10 \mathrm{mV}$ was applied. Electrical insulation was applied to the chamber walls. $S$ was computed as $S=J_{\mathrm{PU}} J_{\mathrm{CC}}\left[\mathrm{m}^{-4}\right]$, where $J_{\mathrm{PU}}$ is the current density vector for the PU couple and $J_{\mathrm{CC}}$ is the current density for the CC couple. ${ }^{25}$ ESI S2 and S3† show simulations of different (i) chamber geometries, (ii) electrode distances and (iii) phantoms (made of stainless steel and acrylic plastic) to assess the suitability of the method for sensing samples with a wide range of electric properties.

\subsection{Design and fabrication of the measurement chamber array}

An array of four chambers (dimensions: $16 \times 19 \times 10 \mathrm{~mm}^{3}$ ) for parallel analysis was micromilled from a polycarbonate substrate having a thickness of $15 \mathrm{~mm}$ (Fig. 2). Within each chamber, eight cylindrical holes (Ø $1.1 \mathrm{~mm}, 2 \mathrm{~mm}$ deep) were 


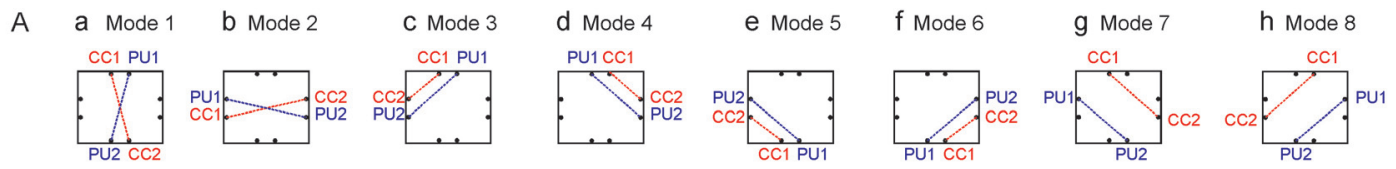

\section{B}
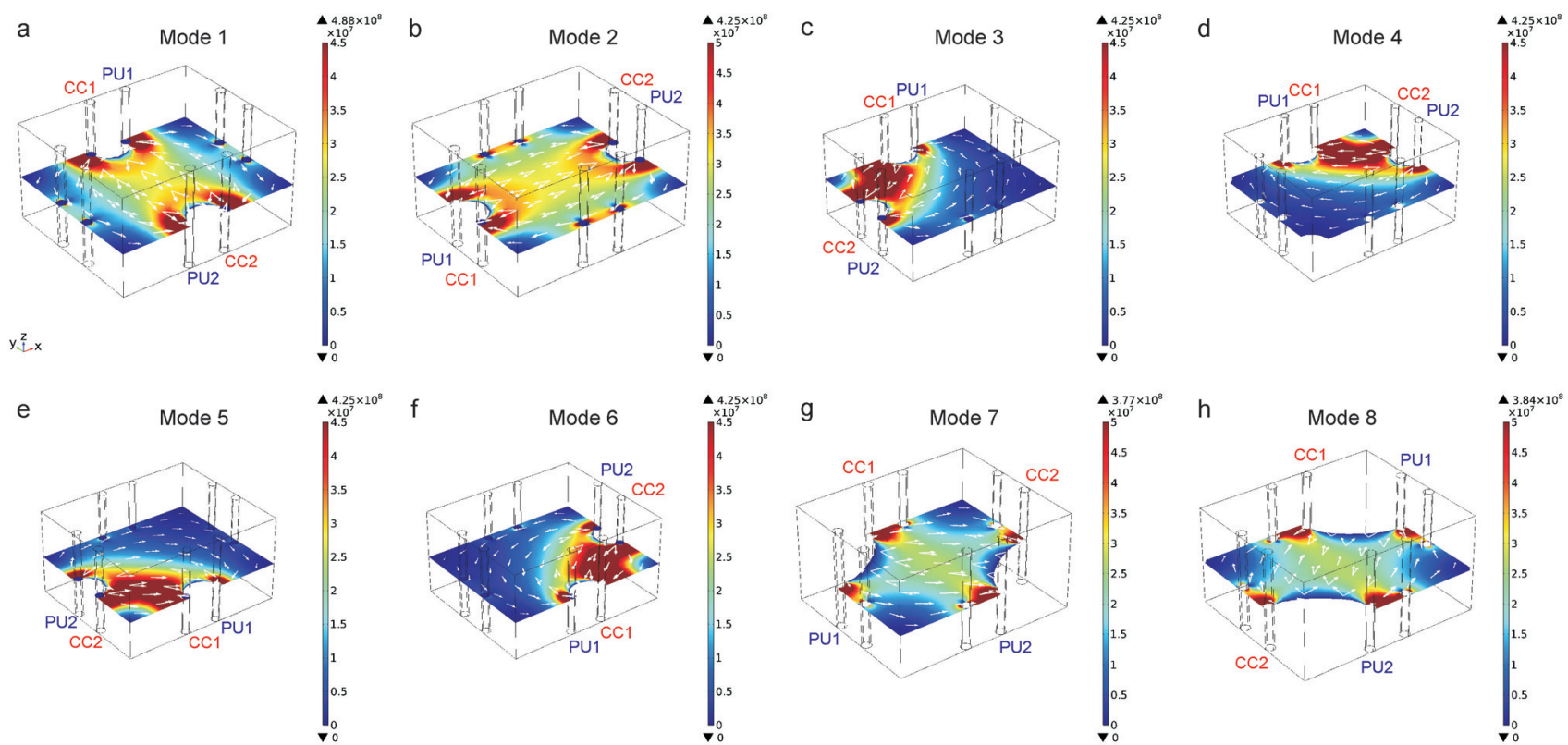

Fig. 1 (A) Schematics of the eight optimised 4T configurations (modes 1-8). CC1 and CC2 form the current-carrying electrode couple and PU1 and PU2 form the voltage pick-up couple. Red and blue dashed lines represent the directions of CC and PU electric fields, respectively. (B) FE simulations for $S\left[\mathrm{~m}^{-4}\right.$ ] distribution (slice view): mode 1 (a), mode 2 (b), mode 3 (c), mode 4 (d), mode 5 (e), mode 6 (f), mode 8 (h). White areas in the horizontal cross-sections are associated with negative sensitivity.
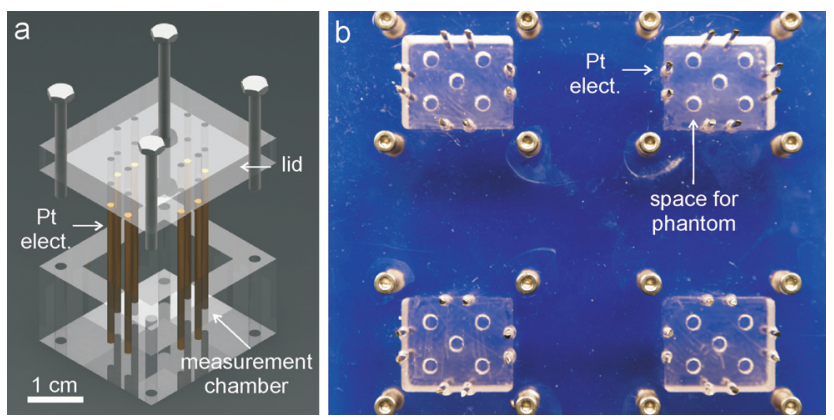

Fig. 2 (a) The measurement chamber design. (b) Photo of the measurement chamber, showing electrode position and openings for phantoms.

drilled along the periphery of the measurement chamber for holding the Pt electrodes $(\varnothing 1 \mathrm{~mm})$ in place. The electrode positioning was determined based on the results of the FE simulation. Pt was chosen as it has been demonstrated to be a suitable biocompatible material for AC applications. ${ }^{27}$ Electrodes were located in couples, $4 \mathrm{~mm}$ apart from each other on each perpendicular side of the chamber (Fig. 2a). A lid for the measurement chamber was fabricated using $5 \mathrm{~mm}$ thick polycarbonate with holes in different positions (Fig. $2 \mathrm{~b}$ ) for place- ment of (i) electrodes and (ii) cylindrical phantoms having different diameters $(2,3,4,5,6,8 \mathrm{~mm})$. Crocodile clips were used for contacting the electrodes to the impedance analyser. Prior to each experiment, the Pt electrodes were cleaned for $10 \mathrm{~min}$ in acetone followed by rinsing with Milli-Q water (Millipore Corporation, Billerica, MA, USA) and potential cycling in $0.1 \mathrm{M} \mathrm{H}_{2} \mathrm{SO}_{4}(-0.4$ to $1.7 \mathrm{~V}$ vs. $\mathrm{Ag} / \mathrm{AgCl}(3 \mathrm{M} \mathrm{KCl})$; approximately 40 cycles at a scan rate of $200 \mathrm{mV} \mathrm{s}^{-1}$ ).

\subsection{Phantom experiments}

The eight simulated modes of $4 \mathrm{~T}$ configuration were validated with phantom experiments using stainless steel and plastic cylinders of increasing dimensions ( $\varnothing 2-8 \mathrm{~mm}$ ) placed in different positions (centre and four corners, Fig. 2b) inside the measurement chambers filled with conductivity standard solution (1.3 $\mathrm{S} \mathrm{m}^{-1}$ Hanna Instruments, cat. 663-5047). A $10 \mathrm{mV} \mathrm{AC}$ potential was applied in the frequency range between $1 \mathrm{kHz}$ and $1 \mathrm{MHz}$ using an impedance analyser SI1260 and a SI1294 impedance interface (Solartron Instruments, Hampshire, UK). For all phantom experiments, characterisation data are presented as an average of three individual experiments in each chamber using fresh solution and rinsing the phantom with Milli-Q water prior to each experiment (average \pm s.e.m., $n=12)$. 


\subsection{Spatial sensing of artificial 3D cell constructs}

Prior to impedance measurements, the chambers were sterilised with $70 \%$ ethanol for $20 \mathrm{~min}$ and allowed to dry in a laminar flow bench. Artificial 3D cell constructs $(\varnothing 4.6 \mathrm{~mm}$ and height $10 \mathrm{~mm}$ ) were prepared by encapsulating $10^{7}$ HepG2 cells (American Type Culture Collection, Rockville, MD, USA) in $5 \% \mathrm{w} / \mathrm{v}$ gelatin (48723 Fluka). $12.5 \%(\mathrm{w} / \mathrm{v})$ gelatin stocks were dissolved in serum-free cell culture medium (Roswell Park Memorial Institute RPMI 1640). The gel was covalently cross-linked using microbial transglutaminase (Activa ${ }^{\circledR} \mathrm{RM}$, Ajinomoto) dissolved in RPMI 1640 containing 10\% fetal bovine serum and $1 \%$ penicillin/streptomycin. Cells were directly mixed with liquid gelatin and cast in a cylindrical mould with a piston for cylinder extrusion after curing ( 4 hours at $37{ }^{\circ} \mathrm{C}$ in a humidified $5 \% \mathrm{CO}_{2}$ incubator). Measurements were performed placing a cylindrical artificial $3 \mathrm{D}$ cell construct in two different positions inside the chamber (centre or top left corner), and $2 \mathrm{~mL}$ of $5 \%$ (w/v) gelatin was cast around and let polymerise for 2 hours. After gelatin polymerisation, the chamber was filled with cell culture medium. EIS spectra were acquired before and after medium addition, as described in Section 2.3. Data for artificial 3D cell constructs were compared with plain $5 \%(\mathrm{w} / \mathrm{v})$ gelatin cylinders surrounded by $2 \mathrm{~mL}$ of $5 \% \mathrm{w} / \mathrm{v}$ gelatin. Measurements were performed in three independent experiments using a different cylinder and filling gelatin (each position tested in duplicate). Data are presented as average \pm s.e.m., $n=6$. Control experiments comprised measurements on $5 \%(\mathrm{w} / \mathrm{v})$ bulk gelatin scaffolds without any added gelatin cylinder. 21 individual experiments were performed (average \pm s.e.m., $n=21$ ) using $5 \%(\mathrm{w} / \mathrm{v})$ bulk gelatin scaffold (three different stock solutions) filling the same volume as in the presence of a gelatin cylinder $\left(16 \times 19 \times 6.6 \mathrm{~mm}^{3}\right)$.

\section{Results and discussion}

\subsection{FE simulation of $S$ distribution}

Martinsen and Grimnes previously illustrated the significance of electrode configuration for impedance measurements focusing on a sub-volume in a physiological 3D sample. ${ }^{21}$ In this study, we optimised eight different modes of $4 \mathrm{~T}$ configuration (Fig. 1A) for spatial sensing in a $3 \mathrm{D}$ environment. The electrode distance was evaluated with respect to the measurement chamber size (ESI S2 $\dagger$ ). To design configurations that individually focus on specific sub-volumes inside the chamber and cover the entire chamber volume (Fig. 1), sites for current injection and voltage measurement were established by minimising negative sensitivity zones. Based on FE simulations, modes 1 and 2 (Fig. 1Ba,b) are expected to have a sensitivity covering the entire chamber volume, however, mainly focusing on the centre. Mode 2, associated with longer current path, results in a higher simulated impedance. Modes 3, 4, 5 and 6 (Fig. 1Bc-f) focus on the corners in a symmetrical fashion, therefore, yielding the same impedance for a homogeneous 3D volume. They show very low sensitivity in the centre. Mode 3, focusing on the top left corner, has $S=0$ at the bottom right corner. Due to symmetry, all the other modes (4-6) have the maximum $S$ in one corner, whereas $S=0$ in the opposite corner (diagonally). Modes 7 and 8 (Fig. 1Bg,h) show symmetrical diagonal current paths through the centre of the chamber, with lower $S$ with respect to modes 1 and 2. They also slightly sense two diagonally located corners and have large zones of negative sensitivity in the two other corners. The eight configurations were further evaluated based on $\mathrm{FE}$ simulation of a metal and plastic object (phantom) inside the chamber (ESI S3†). Results indicate that each mode individually provides $S$ focusing on a specific sub-volume and that they provide information regarding the position of an object in the chamber through the distribution of relative impedance changes in the whole $3 \mathrm{D}$ volume.

\subsection{Phantom experiments}

Phantom experiments were used to verify FE simulations and determine the limit of detection for the sensing method. In impedance measurements, a metal phantom, having a higher conductivity than the surrounding electrolyte medium, decreases the measured impedance in comparison with measurements on the same solution without a phantom. In the case of a nonconductive phantom (plastic object), the effect is the opposite. ${ }^{28}$ This applies to measurements when the phantom is placed in a zone of positive $S$ (Fig. 1B). Fig. 3 and 4 show the impedance spectra obtained when using metal and plastic phantoms of increasing diameter, respectively, placed in the centre of the measurement chamber. The same analysis was performed for $4 \mathrm{~mm}$ diameter phantoms placed in the corners of the chamber. All the experimental results are summarised in ESI S4. $\dagger$

Fig. 3a,b and 4a,b show acquired spectra for modes 1 and 2, respectively, in the presence of a metal or plastic phantom with increasing diameter placed in the centre of the chamber. Corresponding spectra for modes 3 and 7 are shown in Fig. 3c, $\mathrm{d}$ and $4 \mathrm{c}, \mathrm{d}$. A phantom placed in the centre of the chamber is primarily sensed with modes 1,2 and 7, which focus on the entire volume, whereas mode 3 only slightly senses the presence of the phantom. On virtue of symmetry, modes 4-6 (Table $\mathrm{S} 4 \dagger$ ) show exactly the same behaviour as mode 3 . Since mode 3 has weak sensitivity for the centre, the spectrum for the $2 \mathrm{~mm}$ metal phantom (Fig. 3c) fully overlaps with that of the blank. In the case of plastic phantoms, the spectrum for $2 \mathrm{~mm}$ phantom is below the impedance level of the blank, while that for the $3 \mathrm{~mm}$ phantom overlaps with the spectrum of the blank. From Fig. 3 and 4, it can be concluded that our method has the limit of detection of $4 \mathrm{~mm}$ in terms of object dimension regardless of material properties. This corresponds to $16.5 \%$ of the total volume in the centre of the chamber. This applies also to phantoms placed in the measurement chamber corners (Table S4†).

For all modes, the spectra acquired for the different metal phantoms show good discrimination in terms of phantom diameter in the frequency range 100-300 kHz, with mode 2 giving the highest impedance. For plastic phantoms, the impedance 

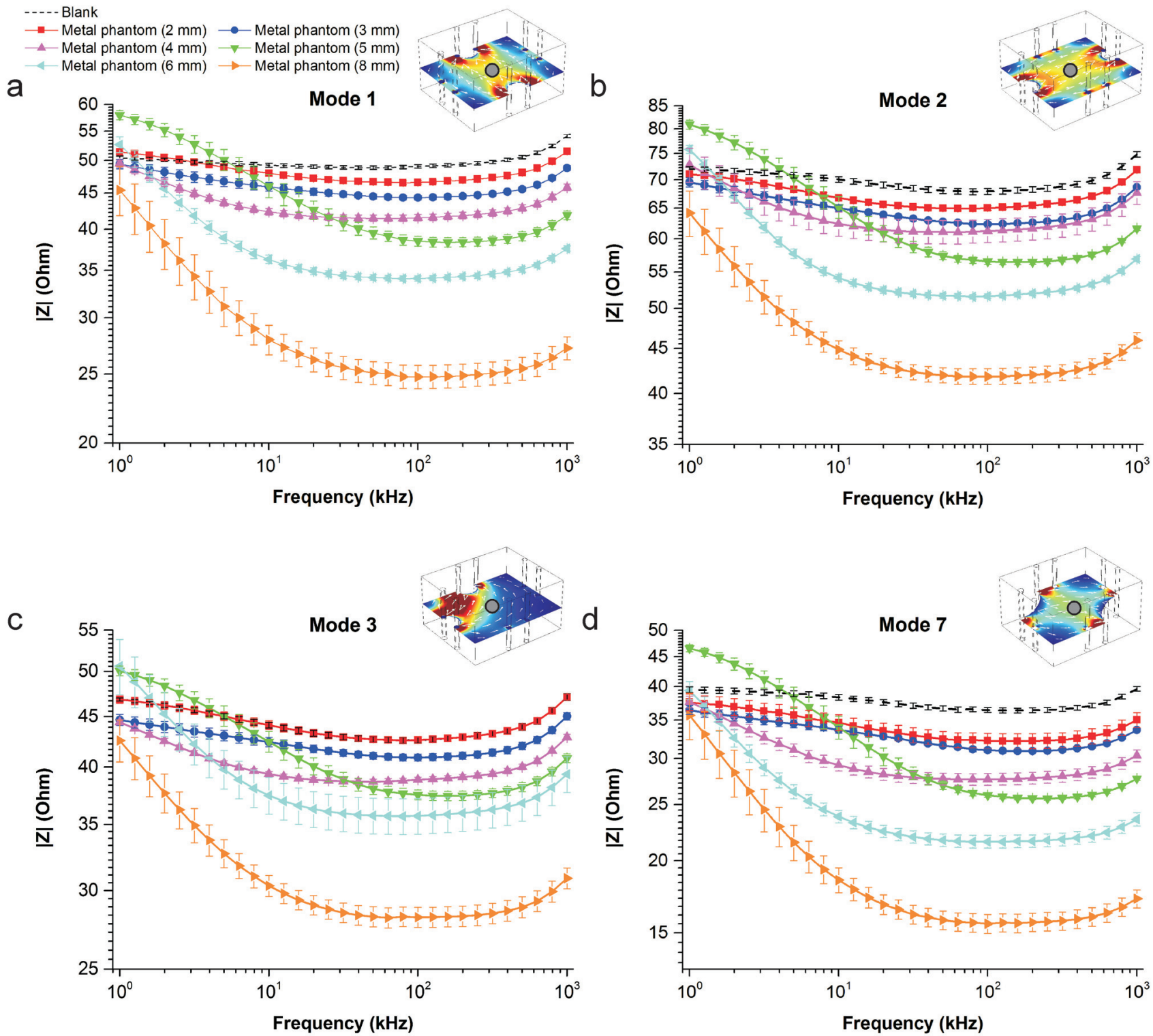

Fig. 3 Characterisation using a metal phantom of increasing diameter $(2-8 \mathrm{~mm})$ placed in the centre of the measurement chamber (indicated by a grey dot) filled with conductivity standard solution: (a) mode 1, (b) mode 2, (c) mode 3, (d) mode 7. Data are compared with spectra for the same solution (blank) and reported as average \pm s.e.m., $n=12$.

magnitude of the spectra acquired for the different modes remains more constant through a wider frequency range. For general analysis of the spectra, $250 \mathrm{kHz}$ is a suitable frequency when comparing the behaviour of different objects in the chambers. This choice of frequency has also the advantage that it is low enough not to be significantly affected by parasitics that appear at higher frequencies (above $300 \mathrm{kHz}$ ).

For a phantom placed in one of the chamber corners, all the modes show good discrimination at $250 \mathrm{kHz}$. All spectra were analysed at this frequency and summarised in Table S4, ESI S4. $\dagger$ Modes 3-6 show the highest $S$ for a phantom placed in the corner where the mode has specific focus according to the FE simulation (Fig. 1Bc-f), with the $S$ being lowest in the opposite corner (diagonally). Modes 1 and 2 do not show any discrimination of phantoms placed in the corners due to their specific focus on the centre of the measurement chamber. Modes 7 and 8 have two diagonal corners where the presence of the phantom can be weakly sensed. However, the spectra acquired for a phantom in either of those corners are overlapping and cannot be discriminated, as expected from the FE analysis (Fig. 1Bg,h). In the two other corners, the presence of the phantom cannot be sensed due to the large zones of negative sensitivity. This represents a situation where the phantom is partially located in the zone of negative and positive sensitivity (depending on its size) which makes it difficult to distinguish the spectra for the phantoms from that of the blank. 


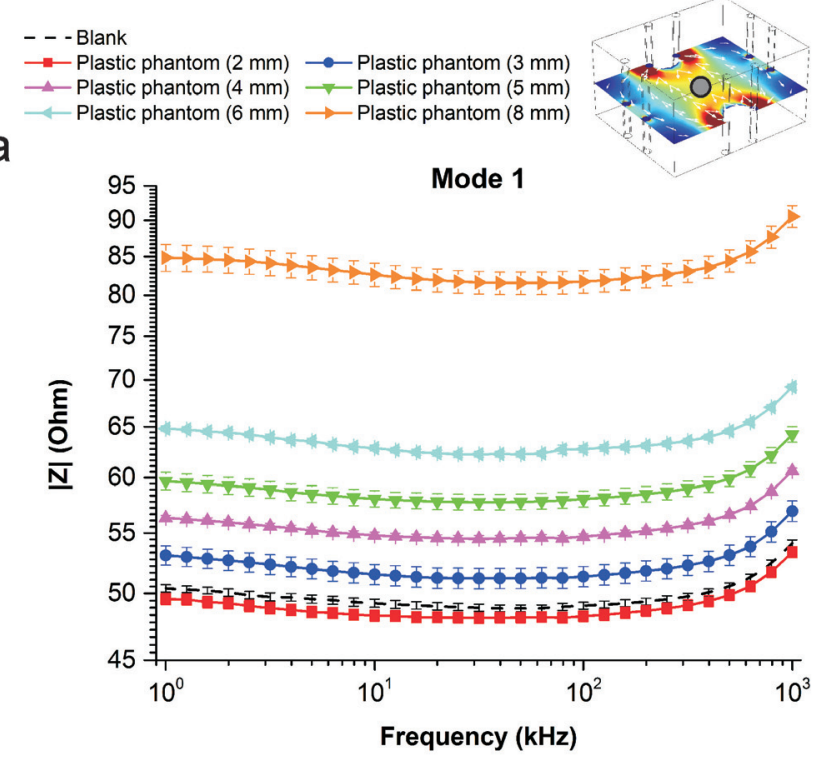

b

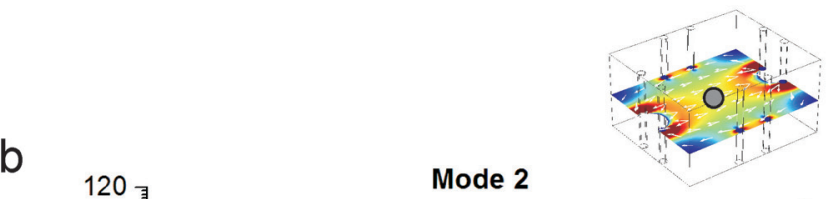

Frequency $(\mathbf{k H z})$
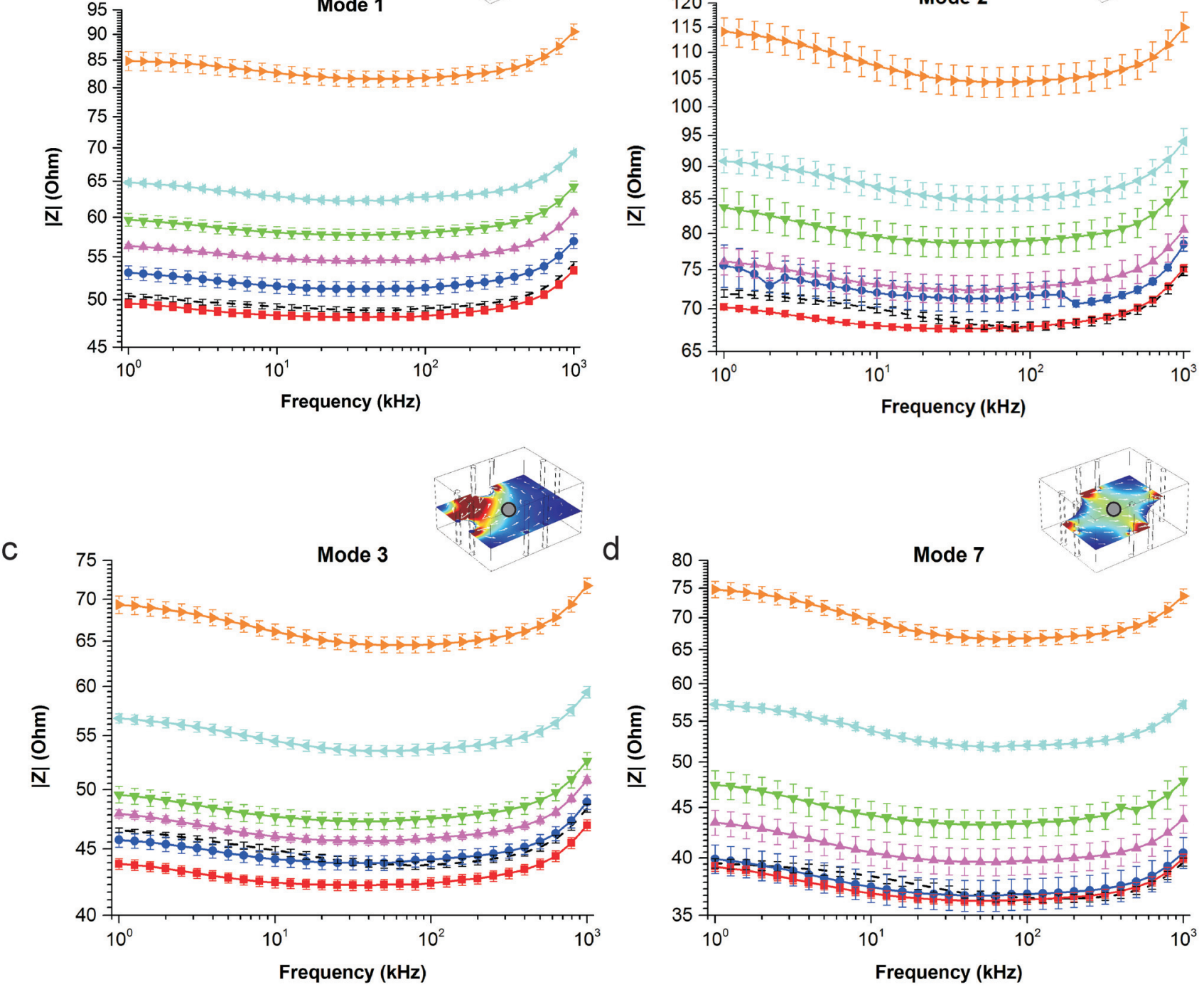

Fig. 4 Characterisation using a plastic phantom of increasing diameter $(2-8 \mathrm{~mm})$ placed in the centre of the measurement chamber (indicated by a grey dot) filled with conductivity standard solution: (a) mode 1, (b) mode 2, (c) mode 3, (d) mode 7. Data are compared with spectra for the same solution (blank) and reported as average \pm s.e.m., $n=12$.

As expected, by increasing the phantom diameter, the absolute variation in measured impedance increased for all eight modes.

\subsection{Spatial sensing of artificial $3 \mathrm{D}$ cell constructs}

The sensing method was further validated with artificial 3D cell constructs using $5 \%(\mathrm{w} / \mathrm{v})$ gelatin cylinders, embedding high density of HepG2 cells to mimic a biological tissue placed in the centre and at the top left corner of a large $5 \%(\mathrm{w} / \mathrm{v})$ bulk gelatin scaffold. The Young's modulus of these enzymatically cross-linked gelatin constructs is approximately $3.6 \mathrm{kPa}$ (data not shown), which is within the stiffness range for soft tissues, such as liver. ${ }^{6,29,30}$

The variability between different bulk gelatin scaffolds was assessed for impedance measurements with the eight different modes after medium addition on top (ESI S5 $\dagger$ ). The s.e.m. were in all cases one order of magnitude lower than the averaged impedance values at $250 \mathrm{kHz}$ (Table S5 $\dagger$ ), indicating the good reproducibility of our method. This also demonstrates that there is low variability between different gelatin stocks and different $5 \%(\mathrm{w} / \mathrm{v})$ bulk gelatin scaffolds. Since modes that focus on the chamber corners show current paths having the same length, they are expected to give the same impedance 
value if the bulk gelatin scaffold is homogeneously distributed in the 3D space, with a smooth top surface. However, we found that the measured impedances were similar for corners on the same side, i.e. the impedance associated to the top left corner (mode 3, $43 \pm 3 \Omega$ ) was similar to the one measured for the bottom left corner (mode 5, $44 \pm 3 \Omega$ ) and the impedance for the top right corner (mode 4, $58 \pm 5 \Omega$ ) was similar to the one for the bottom right corner (mode 6, $56 \pm 5 \Omega$ ). This is probably due to the fact that the measurement chambers were always slightly tilted in the incubator, with slightly more medium covering the left side of each scaffold. Hence, the higher conductivity of cell culture medium ${ }^{31,32}$ above the gelatin provides an additional current path bypassing the gelatin, which decreases the measured overall impedance. Modes 7 and 8, focusing primarily on the chamber centre and slightly on diagonal corners, showed similar values of impedance (37 \pm 3 and $40 \pm 3 \Omega$, respectively).

Fig. 5 shows spatial characterisation using a plain gelatin cylinder placed at the top left corner and in the centre of the bulk gelatin scaffold (Fig. S6.1†). Based on the results reported

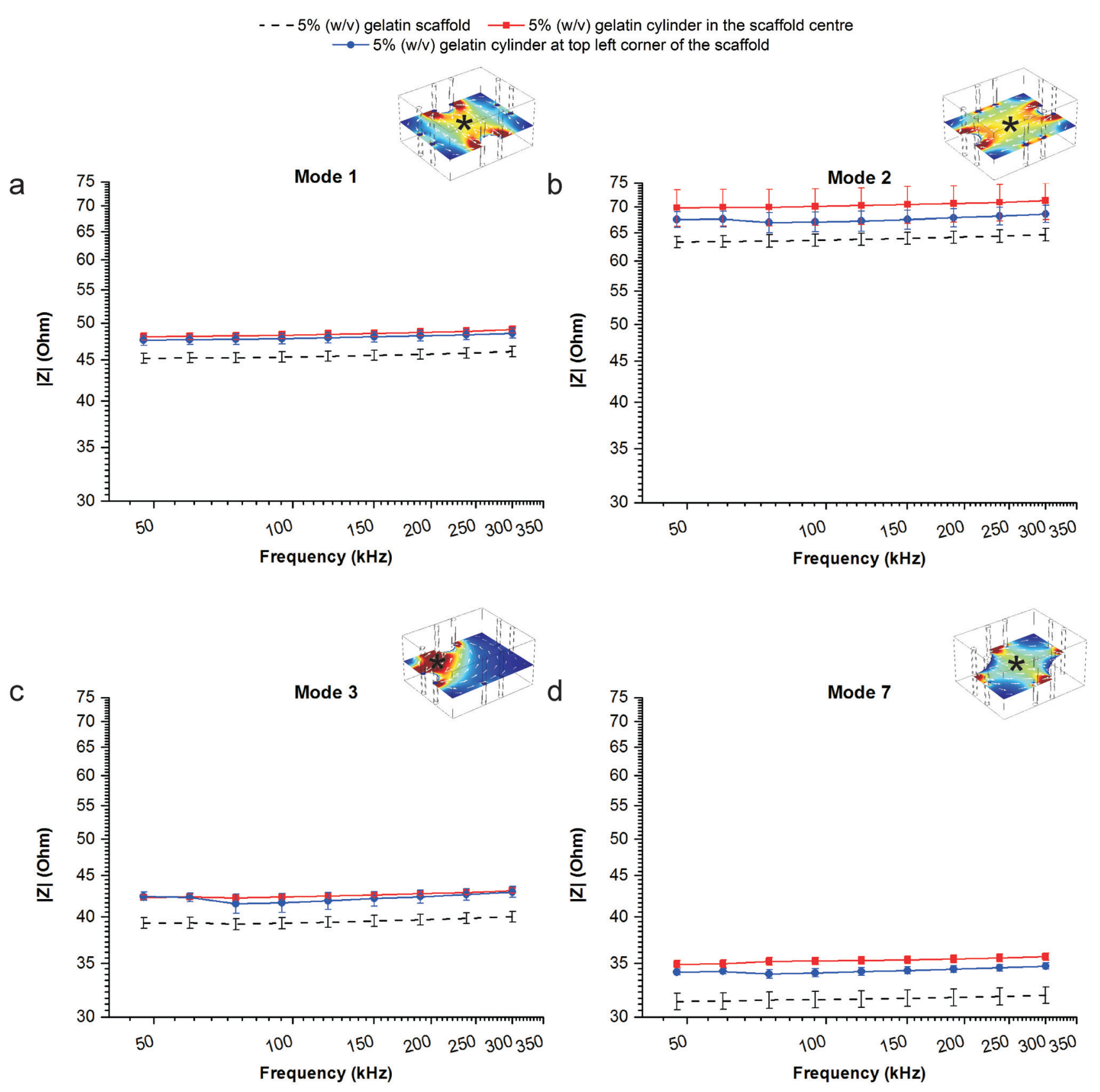

Fig. 5 Sensing of a $5 \%(w / v)$ gelatin cylinder embedded in bulk gelatin scaffold and placed either at the top left corner or in the centre: (a) mode 1 , (b) mode 2, (c) mode 3, (d) mode 7. Data are compared with $5 \%$ (w/v) bulk gelatin scaffold (average \pm s.e.m., $n=21$ ) and reported as average \pm s.e.m., $n=6$. Other modes are shown in Fig. S6.2. $\dagger$ The position of the main focus of each mode is indicated by an asterisk. 
above, measurements were taken after addition of medium on top of the scaffold and the most significant frequency range up to $300 \mathrm{kHz}$ is shown. As expected, the sensing method cannot discriminate between cylinder positions since both cylinder and scaffold are made of the same biomaterial. However, disregarding the measurement mode, the impedance for scaffolds with embedded cylinders is always higher than for bulk gelatin scaffolds. This may be related to surface inhomogeneities originating when casting gelatin around the cylinder or to the possibility that the cylinder becomes slightly more solidified than the bulk gelatin scaffold when this latter is solidifying in the chamber. None of the sensing modes shows any significant difference between the two tested positions (centre and top left corner). However, what was illustrated in the previous paragraphs for metal and plastic phantoms also applies for gelatin cylinders. Mode 1 (Fig. 5a) resulted in lower impedance than mode 2 (Fig. 5b) due to its shorter current path, while modes 7 (Fig. 5d) and 8

$---5 \%$ w/v scaffold $\rightarrow-3 D$ cell construct in the scaffold centre

$\rightarrow 3 \mathrm{D}$ cell construct at the top left corner of the scaffold

a
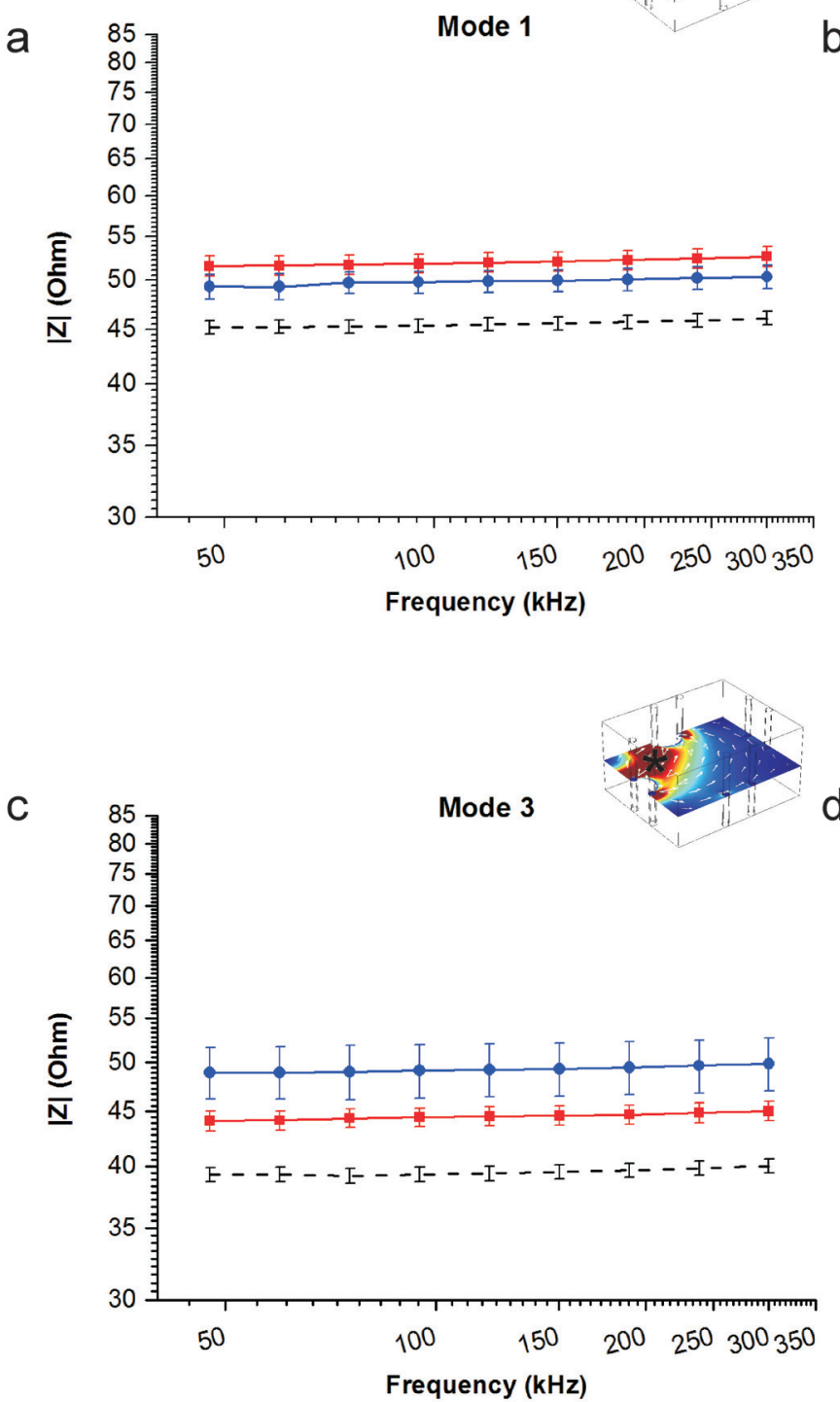

b

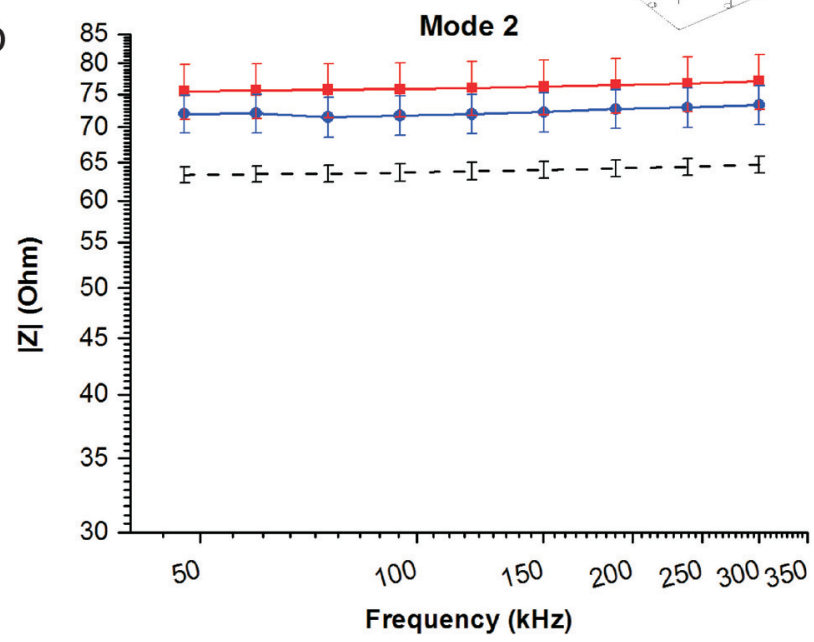

Mode 7
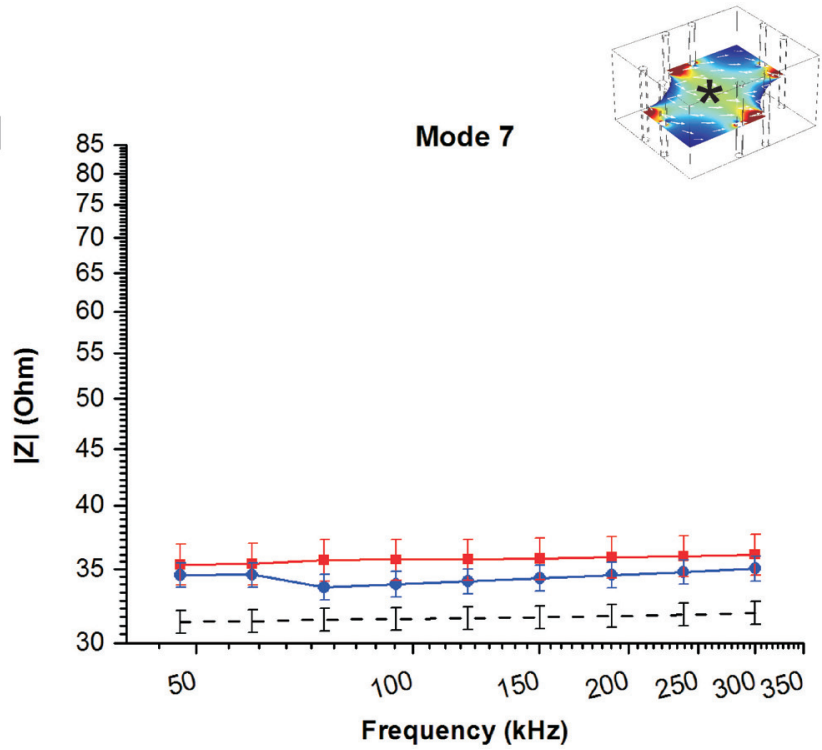

Fig. 6 Sensing of a 5\% (w/v) gelatin cylinder containing $10^{7}$ HepG2 cells as artificial 3D cell construct embedded in bulk gelatin scaffold and placed either at the top left corner or in the centre. Mode 1 (a), mode 2 (b), mode 3 (c), mode 7 (d). Data are compared with $5 \%$ (w/v) bulk gelatin scaffold (average \pm s.e.m., $n=21$ ) and reported as average \pm s.e.m., $n=6$. Other modes are shown in Fig. S6.3. $\dagger$ The position of the main focus of each mode is indicated by an asterisk. 
(Fig. S6.2d†) resulted in similar impedance values as they have equal and symmetrical current paths. Due to the symmetrical current paths, the sensing modes focusing on the corners (mode 3: Fig. 5c; modes 4-6: Fig. S6.2a-c†) gave similar impedance values for a gelatin cylinder in the centre of the measurement chamber. Moreover, since the used gelatin cylinders and the surrounding scaffold had the same material composition, mode 3 did not show any difference in comparison with modes 4-6 when a gelatin cylinder was placed in the top left corner.

Fig. 6 and S6.3† show corresponding data for the artificial cylindrical 3D cell constructs placed at the top left corner and in the centre. For each sensing mode, the $3 \mathrm{D}$ cell constructs showed higher impedance in comparison with the plain gelatin cylinders (control, Fig. 5 and S6.2†). As expected, modes 1 and 2 (Fig. 6a and b) showed higher impedance when the cylinder was placed in the centre and lower impedance when it was placed in the top left corner. The same applies to modes 7 (Fig. 6d) and 8 (Fig. S6.3d $\dagger$ ). The key validation of the sensing method was given by mode 3 (Fig. 6c), resulting in higher impedance when the $3 \mathrm{D}$ cell construct was placed at the top left corner (i.e. the position where it was shown to have the highest $S$ ). The spectrum for the top left corner is clearly separated from the one representing the centre, where mode 3 has a very low $S$. Mode 6 (Fig. S6.3c $\dagger$ ), which has $S=0$ at the top left corner, gives a further validation. In this case, the impedance magnitude of the spectra is considerably higher when the $3 \mathrm{D}$ cell construct was placed in the centre compared to the top left corner. Modes 4 and 5 (Fig. S6.3a,b $\dagger$ ) showed a similar behaviour disregarding the position of the $3 \mathrm{D}$ cell construct.

The s.e.m. for all the acquired spectra in relation to the different modes and positions of the gelatin cylinders (with and without cells) were of the same order of magnitude and slightly below $10 \%$. However, the s.e.m for the plain gelatin cylinders is slightly lower, which can be explained by the fact that the presence of cells increases the inhomogeneity of the cylinders. The appearing differences in s.e.m between the different modes may be derived from minor variations in electrode positioning which causes deviation from perfect symmetry. Additionally, repeated experiments using the same recleaned and reassembled electrodes in the measurement chambers may affect the electrode shape, therefore also contributing to differences between experiments.

The presented method allows addressing the detection of different sample sub-volumes in a biologically relevant 3D environment. Such method responds to the spatial distribution of cell aggregates in a biocompatible scaffold for tissue engineering. The simulation of the sensitivity field distribution related to each sensing mode provides qualitative information that describes its focus on a specific 3D sub-volume and its weight on the total measured impedance. Hence, the measured impedance using the different sensing modes can be applied in tissue engineering applications to obtain insight about proliferation and accumulation of cells in specific subvolumes of a large 3D scaffold. This may be also applied for scaffold characterisation in terms of structural features (e.g. channels mimicking vascularisation) and potentially used for co-cultures of different cell types.

\section{Conclusions}

Several electrode combinations (sensing modes) can be used for multiplexed 4-terminal (4T) impedance sensing to provide spatial information of objects placed in a biologically relevant $3 \mathrm{D}$ environment, which is compatible with tissue engineering applications. The results presented here demonstrate the potential and suitability of our impedance-based sensing method for future monitoring of tissue engineering processes, such as the formation of bioartificial organs. Our method is a first step towards miniaturisation of impedance detection to enable on-line monitoring with 3D spatial resolution and, provides the fundamental basis for reconstruction of images related to cell distribution in a $3 \mathrm{D}$ environment using several multiplexed measurements.

\section{Acknowledgements}

This study and the Ph.D. fellowship of C. Canali were financially supported by the EU-funded project NanoBio4Trans (grant no. 304842).

\section{References}

1 Y. Huang, X. Wang, F. F. Becker and P. R. C. Gascoyne, Biophys. J., 1997, 73, 1118-1129.

2 R. Pethig and D. B. Kell, Phys. Med. Biol., 1987, 32, 933-970.

3 H. P. Schwan and S. Takashima, Bull. Inst. Chem. Res. Kyoto Univ., 1991, 69, 459-475.

4 J. P. Frampton, M. R. Hynd, J. C. Williams, M. L. Shuler and W. Shain, J. Neural Eng., 2007, 4, 399-409.

5 C. Hildebrandt, H. Büth, S. Cho, Impidjati and H. Thielecke, J. Biotechnol., 2010, 148, 83-90.

6 C. Canali, A. Heiskanen, H. B. Muhammad, P. Høyum, F.-J. Pettersen, M. Hemmingsen, A. Wolff, M. Dufva, $\varnothing$. G. Martinsen and J. Emnéus, Biosens. Bioelectron., 2015, 63, 72-79.

7 C. Canali, S. Mohanty, A. Heiskanen, H. B. Muhammad, Ø. G. Martinsen, M. Dufva, A. Wolff and J. Emnéus, Electroanalysis, 2015, 27, 193-199.

8 H.-G. Jahnke, A. Heimann, R. Azendorf, K. Mpoukouvalas, O. Kempski, A. a Robitzki and P. Charalampaki, Biosens. Bioelectron., 2013, 46, 8-14.

9 H. P. Schwan, Adv. Biol. Med. Phys., 1957, 5, 147-209.

10 I. Giaever and C. R. Keese, Proc. Natl. Acad. Sci. U. S. A., 1984, 81, 3761-3764.

11 A. M. Greiner, B. Richter and M. Bastmeyer, Macromol. Biosci., 2012, 12, 1301-1314.

12 C. Moll, J. Reboredo, T. Schwarz, A. Appelt, S. Schürlein, H. Walles and S. Nietzer, J. Vis. Exp. JoVE, 2013, 2-7. 
13 B. M. Baker and C. S. Chen, J. Cell Sci., 2012, 125, 30153024.

14 K. F. Lei, M.-H. Wu, C.-W. Hsu and Y.-D. Chen, Biosens. Bioelectron., 2014, 51, 16-21.

15 D. Holder, in Electrical impedance tomography, IOP Publishing, London, UK, 1st edn, 2005, pp. 411-422.

16 D. Holder, in Electrical impedance tomography, IOP Publishing, London, UK, 1st edn, 2005, pp. 423-449.

17 P. Kauppinen, J. Hyttinen and J. Malmivuo, Int. J. Bioelectromagn., 2006, 7, 344-347.

18 A. McEwan, G. Cusick and D. S. Holder, Physiol. Meas., 2007, 28, S197-S215.

19 D. B. Geselowitz, IEEE Trans. Biomed. Eng., 1971, BME-18, 38-41.

20 J. Malmivuo, J. Phys. Conf. Ser., 2010, 224, 012001.

21 Ø. G. Martinsen and S. Grimnes, in Bioimpedance and Bioelectricity Basics, ed. Ø. G. Martinsen and S. Grimnes, Academic Press, 2nd edn, 2008, pp. 127-152.
22 S. Grimnes and $\varnothing$. G. Martinsen, J. Phys. D: Appl. Phys., 2007, 40, 9-14.

23 H. P. Schwan, Ann. N. Y. Acad. Sci., 1968, 148, 191-209.

24 B. H. Brown, A. J. Wilson and P. Bertemes-Filho, Electron. Lett. , 2000, 36, 5-6.

25 F.-J. Pettersen and J. O. Høgetveit, J. Electr. Bioimpedance, 2011, 2, 13-32.

26 Ø. G. Martinsen and S. Grimnes, in International Federation for Medical and Biological Engineering, 2009, pp. 1078-1079.

27 H. P. Schwan, Biophysik, 1966, 3, 181-201.

28 S. Grimnes and Ø. G. Martinsen, Encycl. Biomed. Eng., 2006, 1-9.

29 P. a Janmey and R. T. Miller, J. Cell Sci., 2011, 124, 9-18.

30 G. C. Reilly and A. J. Engler, J. Biomech., 2010, 43, 55-62.

31 H. Hsu, A. T. Ohta, P.-Y. Chiou, A. Jamshidi, S. L. Neale and M. C. Wu, Lab Chip, 2010, 10, 165-172.

32 C. Canali, H. B. Muhammad, Ø. G. Martinsen and A. Heiskanen, Sens. Actuators, B, 2015, 544-550. 